\title{
Regional homogeneity, resting-state functional connectivity and amplitude of low frequency fluctuation associated with creativity measured by divergent thinking in a sex-specific manner
}

\author{
Hikaru Takeuchi ${ }^{\mathrm{a}, *}$, Yasuyuki Taki ${ }^{\mathrm{a}, \mathrm{b}, \mathrm{c}}$, Rui Nouchi ${ }^{\mathrm{d}, \mathrm{e}, \mathrm{f}}$, Ryoichi Yokoyama ${ }^{\mathrm{g}}$, Yuka Kotozaki ${ }^{\mathrm{h}}$, \\ Seishu Nakagawa $a^{\mathrm{i}, \mathrm{j}}$, Atsushi Sekiguchi ${ }^{\mathrm{b}, \mathrm{i}, \mathrm{k}}$, Kunio Iizuka, Yuki Yamamoto ${ }^{\mathrm{i}}$, Sugiko Hanawa ${ }^{\mathrm{i}}$, \\ Tsuyoshi Araki ${ }^{\mathrm{f}}$, Carlos Makoto Miyauchi ${ }^{\mathrm{m}}$, Takamitsu Shinada ${ }^{\mathrm{i}}$, Kohei Sakaki ${ }^{\mathrm{i}}$, \\ Takayuki Nozawa $^{n}$, Shigeyuki Ikeda ${ }^{n}$, Susumu Yokota ${ }^{a}$, Magistro Daniele ${ }^{\circ}$, Yuko Sassa ${ }^{a}$, \\ Ryuta Kawashima, a,f,i \\ a Division of Developmental Cognitive Neuroscience, Institute of Development, Aging and Cancer, Tohoku University, Sendai, Japan \\ b Division of Medical Neuroimaging Analysis, Department of Community Medical Supports, Tohoku Medical Megabank Organization, Tohoku University, \\ Sendai, Japan \\ ${ }^{\mathrm{c}}$ Department of Radiology and Nuclear Medicine, Institute of Development, Aging and Cancer, Tohoku University, Sendai, Japan \\ d Creative Interdisciplinary Research Division, Frontier Research Institute for Interdisciplinary Science, Tohoku University, Sendai, Japan \\ e Human and Social Response Research Division, International Research Institute of Disaster Science, Tohoku University, Sendai, Japan \\ ${ }^{\mathrm{f}}$ Smart Ageing International Research Center, Institute of Development, Aging and Cancer, Tohoku University, Sendai, Japan \\ ${ }^{\mathrm{g}}$ School of Medicine, Kobe University, Kobe, Japan \\ h Division of Clinical research, Medical-Industry Translational Research Center, Fukushima Medical University School of Medicine, Fukushima, Japan \\ i Department of Functional Brain Imaging, Institute of Development, Aging and Cancer, Tohoku University, Sendai, Japan \\ ${ }^{\mathrm{j}}$ Department of Psychiatry, Tohoku Pharmaceutical University, Sendai, Japan \\ ${ }^{\mathrm{k}}$ Department of Adult Mental Health, National Institute of Mental Health, National Center of Neurology and Psychiatry, Tokyo, Japan \\ ${ }^{1}$ Department of Psychiatry, Tohoku University Graduate School of Medicine, Sendai, Japan \\ ${ }^{\mathrm{m}}$ Graduate School of Arts and Sciences, Department of General Systems Studies, The University of Tokyo, Tokyo, Japan \\ ${ }^{n}$ Department of Ubiquitous Sensing, Institute of Development, Aging and Cancer, Tohoku University, Sendai, Japan \\ - School of Electronic, Electrical and Systems Engineering, Loughborough University, England, UK
}

\section{A R T I C L E I N F O}

Keywords:

Resting state

Regional coherence

Creativity

Divergent thinking

Anterior temporal lobe

Sex difference

Functional connectivity

\begin{abstract}
A B S T R A C T
Brain connectivity is traditionally thought to be important for creativity. Here we investigated the associations of creativity measured by divergent thinking (CMDT) with resting-state functional magnetic imaging (fMRI) measures and their sex differences. We examined these relationships in the brains of 1277 healthy young adults. Whole-brain analyses revealed a significant interaction between verbal CMDT and sex on (a) regional homogeneity within an area from the left anterior temporal lobe (b) on the resting state functional connectivity (RSFC) between the mPFC and the left inferior frontal gyrus and (c) on fractional amplitude of low frequency fluctuations (fALFF) in several distinct areas, including the precuneus and middle cingulate gyrus, left middle temporal gyrus, right middle frontal gyrus, and cerebellum. These interactions were mediated by positive correlations in females and negative correlations in males. These findings suggest that greater CMDT in females is reflected by (a) regional coherence (regional homogeneity) of brain areas responsible for representing and combining concepts as well as (b) the efficient functional connection (RSFC) between the key areas for the default state of cognitive activity and speech production, and (c) greater spontaneous neural activity (fALFF) during the resting of brain areas involved in frontal lobe functions, default cognitive activities, and language functions. Furthermore, these findings suggest that the associations between creativity and resting state brain connectivity patterns are different between males and females.
\end{abstract}

\footnotetext{
* Corresponding author.

E-mail address: takehi@idac.tohoku.ac.jp (H. Takeuchi).
} 


\section{Introduction}

A common definition of creativity is the ability to produce work that is both novel and useful within a certain social context (Runco and Jaeger, 2012; Stein, 1953). The most common measure to evaluate creativity is divergent thinking (DT) ["information retrieval and the call for a number of varied responses to a certain item" (Guilford, 1967)]. Indeed, performance on DT tasks can predict real-life creative achievement (for the meta analysis, see Kim, 2008).

The importance of brain connectivity for creativity has been predicted theoretically and demonstrated experimentally. Greater brain connectivity has been suggested to be essential for creativity because of the theoretical importance of combining disparate information (Heilman et al., 2003). Greater creativity is associated with the microstructural properties of widespread white matter areas (Takeuchi et al., 2010b) and greater functional connectivity between different brain areas during creative tasks (for a review of recent research, see Szameitat et al., 2002).

Recent studies have also shown unique associations between creativity measured by divergent thinking (CMDT) and resting-state functional connectivity (RSFC) for different brain regions. We have suggested that cognitive processes ongoing during rest, such as imagination, daydreaming, fantasy, and attentiveness to one's own feelings as well as the neural bases underlying these cognitive activities are shown or considered to be important for creativity (for summary, see Takeuchi et al., 2012). Previous studies using large sample sizes showed that greater CMDT is associated with greater RSFC between the key nodes of the default mode network (DMN; the network that is active at rest) (Takeuchi et al., 2012) as well as between the medial prefrontal cortex (mPFC), one of the key nodes of the DMN, and the language-related area of the left middle temporal gyrus (Wei et al., 2013).

Recent advances in analysis of resting-state functional magnetic resonance imaging (rsfMRI) have made it possible to reveal new aspects of resting-state brain activity (Chao-Gan and Yu-Feng, 2010). One such advance is fractional amplitude of low frequency fluctuation (fALFF), which is thought to indicate the magnitude of neural activity during rest (Chao-Gan and Yu-Feng, 2010; Zang et al., 2007) and a direct index of the spontaneous signal fluctuations underlying RSFC. Another measure is degree centrality (DC), which reflects the number of instantaneous functional connections between a region and the rest of the brain (Buckner et al., 2009). This measure is suggested to reflect how strongly a given region (node) acts as a cortical "hub" (Buckner et al., 2009), and thus, to reflect how strongly the node influences other brain regions and integrates information from functionally segregated regions. The third measure is regional homogeneity. This evaluates "the similarity of time series of a given voxel to those of its nearest voxels in a voxel-wise way based on the assumption that a voxel was temporally similar to those of its neighbors" (Yao et al., 2009). Using this method, we can measure the temporal homogeneity of regional BOLD signals, which can reflect the temporal homogeneity of neural activity and thus local connectivity of synchronization. These measures have been used to reveal the neural correlates of basic higher-order cognitive abilities such as intelligence, working memory, and attention (Cole et al., 2012; Takeuchi et al., 2015a; Tian et al., 2012; Xu et al., 2014). Given the extent brain connectivity and information integration as well as cognitive processes during rest have been shown and suggested to be important for creativity, we predict associations between CMDT and these neural mechanisms.

Sex differences in creative cognition have garnered attention in the field of cognitive science. The robust sex differences of divergent thinking scores are not consistently observed (Baer and Kaufman, 2008); however, a study has reported that a stronger positive association between creativity and psychopathology is observed in men than in women (Martín-Brufau and Corbalán, 2016). A review has concluded that declarative memory-related regions were strongly activated in men during divergent thinking, whereas regions involved in the theory of mind and self-referential processing were more activated in women (Abraham et al., 2014). Taken together, these studies have suggested the existence of sex differences in the neurocognitive mechanisms of divergent thinking. In addition, a previous study showed sex differences in the structural connectivity correlates of CMDT (Ryman et al., 2014). Similarly, our recent large-scale study (Takeuchi et al., in press; the subjects of this previous study and in the present study are the same, except for 59 subjects whose rsfMRI scans were unavailable) demonstrated a positive association between the CMDT and regional white matter volume (rWMV) across widespread white matter areas, including the temporal lobe, in females only.

Despite the attention CMDT has received in neuroimaging studies, no study has investigated DC/fALFF correlates of CMDT. A previous study has reported a negative association between CMDT and regional homogeneity in the precuneus (Chen et al., 2015). In addition, sex differences between DC, fALFF, regional homogeneity, RSFC, and correlates of CMDT have not been investigated. The purpose of this study was to investigate these issues.

As has been partly described, RSFC involving areas of the DMN and middle temporal gyrus were associated with verbal CMDT in a large sample (Takeuchi et al., 2012; Wei et al., 2013). Furthermore, the key nodes of the DMN, such as the precuneus and MPFC, are thought to be important hubs. Thus, we hypothesized that the DC, and regional homogeneity of these areas are associated with CMDT. Given the aforementioned (a) sex differences in the psychological correlates of CMDT (Martín-Brufau and Corbalán, 2016), (b) sex differences in brain areas that are recruited during CMDT (Abraham et al., 2014), and (c) recent reports on the existence of female-specific associations of CMDT and brain structural connectivity (Ryman et al., 2014; Takeuchi et al., in press), we expected to observe sex-specific (particularly female-specific) associations between connectivity measures and CMDT. Given the significant associations between CMDT and RSFC with the mPFC, a key node of the DMN (Takeuchi et al., 2012), we focused on the mPFC as a seed region for our RSFC analyses. We additionally investigated the associations of CMDT and fALFF, a direct index of the spontaneous signal fluctuations underlying RSFC as well as their sex differences to reveal the nature of the associations between resting state brain connectivity measures and CMDT.

\section{Methods}

\section{Subjects}

The present study, which is a part of an ongoing project to investigate the association among brain imaging, cognitive function, and aging, included 1277 healthy, right-handed individuals (732 men and 545 women). The mean age of the subjects was 20.8 years [standard deviation (SD): 1.8; range: 18-27 years]. All subjects were college, university, postgraduate, or graduate students from the previous year. All had normal vision and no history of neurological or psychiatric illness. Handedness was evaluated using the Edinburgh Handedness Inventory (Oldfield, 1971). Subject characteristics, recruitment, and exclusion criteria are provided in the Supplemental methods. Written informed consent was obtained from each subject for their participation in this project. All study procedures were approved by the Ethics Committee of Tohoku University.

Subjects were instructed to get sufficient sleep, maintain their conditions, eat sufficient breakfast, and to consume their normal amounts of caffeinated foods and drinks in the day of cognitive tests and MRI scans. In addition, subjects were instructed to avoid alcohol the night before the assessment.

The sex ratio in this study sample was unbalanced because the main participant pool was also unbalanced as females were less available. 


\section{Psychological measurements}

The methods outlined here are reproduced from our previous studies (Takeuchi et al., 2011a, page 2; 2011b, page 682; Takeuchi et al., 2012, pages 2923-2924; Takeuchi et al., 2010a, pages 579-580; 2010b, pages 12-13; Takeuchi et al., 2015b pages 3-4) that used exactly the same measures in the same way.

The S-A creativity test (Society_For_Creative_Minds, 1969) was used to assess CMDT. J.P. Guilford generated the draft plan of this test. $\mathrm{He}$ also supervised the development of the test (Society_For_Creative_Minds, 1969). The test was standardized for Japanese speakers (Society_For_Creative_Minds, 1969).

The test is used to evaluate verbal CMDT (Society_For_Creative_Minds, 1969), and it involves three types of tasks. The practice (and real) tasks are administered in the following order: (1) practice of the first task (2 min), (2) the first task (5 min), (3) practice of the second task (2 min), (4) the second task (5 min), (5) practice of the third task (2 min), and (6) the third task (5 min). Each task involves two questions. In total, the test takes $30 \mathrm{~min}$. This test was administered in a group setting. The first task requires subjects to generate unique ways of using typical objects (e.g., "Other than reading, how can we use newspapers?" An example answer is "We can use them to wrap things."). The second task requires subjects to imagine desirable functions of ordinary objects (e.g., "What are the characteristics of a good TV? Write down as many characteristics as possible." An example answer is "A TV can receive broadcasts from all over the world."). The third task requires subjects to imagine the consequences of "unimaginable things" happening (e.g., "What would happen if all the mice in the world disappeared?" An example answer is "The world would become more hygienic."). Each task requires subjects to generate as many answers as possible. Notably, these tasks correspond to the three tasks (unusual use, product improvement, and just suppose) of the Torrance tests of creative thinking (TTCT; Torrance, 1966), which are used in multiple countries. The S-A creativity test provides a total score, which was used in this study, as well as scores for the following dimensions of the creative process: (a) Fluency: Fluency is measured by the number of relevant responses to questions and is related to the ability to produce and consider several alternatives. Fluency scores are determined by the total number of questions answered after excluding inappropriate responses or responses that are difficult to understand. (b) Flexibility: Flexibility is the ability to produce responses from a wide perspective. Flexibility scores are determined by the sum of the (total) number of category types to which the responses are assigned based on a criteria table or an almost equivalent judgment (when the answer is not in the criteria table, the raters score their answers based on the subjective criteria that is similar to criteria of the criteria table). (c) Originality: Originality is the ability to produce ideas that differ from those of others. Originality scoring is based on the sum of idea categories that are weighted based on a criteria table or an almost equivalent judgment. (d) Elaboration: Elaboration is the ability to produce detailed ideas (Society for Creative Mind, 1969). Elaboration scores are determined by the sum of responses that are weighted based on a criteria table or an almost equivalent judgment. These four dimensions correspond to the same concepts as those of the Torrance tests of creative thinking (TTCT; Torrance, 1966).

The total score is the sum of the originality score and that of elaboration in the version of the S-A creativity test (Society_For_Creative_Minds, 1969) used here (i.e., the total score is officially stipulated as the sum of the originality and elaboration scores). This is because the Fluency and Flexibility scores are strongly correlated with those of Elaboration (Society_For_Creative_Minds, 1969). Scoring of the tests was performed by the Tokyo Shinri Corporation.

The analysis was limited to the total score, and it did not include the score for each dimension. This is because in this test the score of each dimension is highly correlated with the total score and with those of other dimensions (Takeuchi et al., 2010b). This phenomenon is consistent with another similar divergent thinking test (Heausler and Thompson, 1988), namely TTCT (Torrance, 1966). Heausler and Thompson (1988) concluded that the correlations among the subscales in TTCT are so high that each subscale could not meaningfully provide dissociated information. Treffinger (1985) also warned that separate interpretations of TTCT subscores should be avoided. Consistent with this notion, a previous study (Chávez-Eakle et al., 2007) that investigated the association between regional cerebral flow (rCBF) and each dimension revealed that different dimensions were correlated with rCBF in similar regions. The description outlined in this paragraph was reproduced from our previous study (Takeuchi et al., 2015b). Here the following average four subscores were obtained: fluency, 34.9; flexibility, 25:3; elaboration, 30.0; and originality, 8.3. The simple correlation coefficients among the subscores included 0.87 for fluency and flexibility, 0.88 for fluency and elaboration, 0.605 for fluency and originality, 0.79 for flexibility and elaboration, 0.72 for flexibility and originality, and 0.57 for elaboration and originality. While the correlations among the subscales were generally very high, originality was distinct and correlations between originality and other subscales are slightly lower than other correlations. And other subscale scores are about 3 times higher than originality scores. Thus, the total score (elaboration+originality) is similar to the sum of all the normalized subscores.

However, as aforementioned, although it was recommended that the use of the subscores be avoided, these subscores have been widely used in the field. Therefore, we provided the results of analyses using these subscores instead of total scores in Supplemental methods, Supplemental results, and Supplemental Fig. 1-7. In brief, significant results or tendency of results of imaging analyses of these subscores, included the areas that are similar to the areas of the significant results of imaging analyses of total scores which were provided in Results section of the main text (below).

Please refer to the appendix of our previous study (Takeuchi et al., 2010a) for a sample and the manner in which the tests were scored.

For the information of the external validity of this scale, we quote our previous study (Takeuchi et al., 2010a, page 579). S-A creativity test scores are significantly correlated with various other external measures, such as various personality factors and problem-solving abilities in daily life, suggesting its ability to predict performance in everyday situations (Shimonaka and Nakazato, 2007). Furthermore, SA creativity test scores are significantly correlated with the frequency of visual hypnagogic experiences, which in turn are correlated with the vividness of mental imagery and neuroticism (Watanabe, 1998).

In addition, Raven's Advanced Progressive Matrix was used to assess intelligence and adjust for the effect of general intelligence on resting-state fMRI measures (Song et al., 2008). For additional test details, please see the Supplemental methods.

\section{Image acquisition}

The descriptions in this subsection were mostly reproduced from our previous study using the exactly the same methods (Takeuchi et al., 2015a). All MRI data acquisition was conducted with a 3-T Philips Achieva scanner. As in our previous study (Takeuchi et al., 2012), 34 transaxial gradient-echo images $(64 \times 64$ matrix, $\mathrm{TR}=2000 \mathrm{~ms}$, $\mathrm{TE}=30 \mathrm{~ms}$, flip angle $=70^{\circ}, \mathrm{FOV}=24 \mathrm{~cm}$, slice thickness $=3.75 \mathrm{~mm}$ ) covering the entire brain were acquired using an echo planar sequence for resting-state fMRI analyses. For this scan, 160 functional volumes were obtained while subjects were resting (resulting in a scan length of $5 \mathrm{~min} 20 \mathrm{~s}$ ). During the resting-state scanning, the subjects were instructed to keep still with their eyes closed, as motionless as possible, and not to sleep and not to think about anything in particular, as has been done similarly (Damoiseaux et al., 2006; Greicius et al., 2003).

To avoid motions, pads and magic tapes were used and subjects 
were given thorough instructions to prevent motion during the scan and explanations as to why motions are not preferable as well as the instruction that excessive motion would lead to the re-scan.

We excluded one subject from the current analyses because of excessive motion $[<3.75 \mathrm{~mm}$ (voxel size of one voxel)] during the scan from the entire sample population whose data related to this study was collected. The subjects were young adults and the scan did not last long.

Before the rsfMRI scan, BOLD images were obtained during the $\mathrm{N}$ back working memory task. For all subjects, task-related fMRI scans were not run immediately before the rsfMRI scans, but rather rsfMRI scans were always taken just after the structural scan. Diffusionweighted data were acquired using a spin-echo EPI sequence. From the collected images, fractional anisotropy (FA) and mean diffusivity (MD) maps of diffusion tensor images were constructed (Takeuchi et al., 2011d). These images were used in the normalization procedures as described below.

\section{Pre-processing of functional imaging data}

The preprocessing of imaging data was performed using SPM8 implemented in MATLAB and SPM8's extension software Data Processing Assistant for Resting-state fMRI (DPARSF) [part of the toolbox for Data Processing and Analysis of Brain Imaging (DPABI) (http://rfmri.org/dpabi)(Yan et al., 2016)].

Briefly, rsfMRI BOLD images were non-linearly registered to the BOLD image of the N-back working memory task, and were then coregistered to the $b=0$ image of the diffusion-weighted images (together with rsfMRI BOLD images). $b=0$ images were aligned with the FA image and MD map. Subsequently, all images were normalized using a previously validated two-step new segmentation algorithm of diffusion images and the previously validated diffeomorphic anatomical registration through the exponentiated lie algebra-based registration process (Takeuchi et al., 2013) with DTI images. The voxel size of normalized rsfMRI BOLD images was $3.75 \times 3.75 \times 3.75 \mathrm{~mm}^{3}$. From these images, whole-brain, white matter, and cerebrospinal fluid (CSF) masks were created. See the Supplemental methods for the details and rationale behind these procedures.

The following descriptions of fALFF analyses and DC analyses were mostly reproduced from our previous study using identical methods (Takeuchi et al., 2015a). The normalized series of BOLD images were processed by DPARSF for individual level analysis. First, 26 nuisance covariates (including the mean timecourse of signals from the voxels within the white matter mask, the mean timecourse of signals from the voxels within the CSF mask, and Friston 24 motion parameters. The Friston 24-parameter model (i.e., six head motion parameters, six head motion parameters one time point before, and the 12 corresponding squared items) (Friston et al., 1996) was used to regress out head motion effects. Recent work indicates that regressing out Friston 24parameters is more effective than other movement correction methods, such as correction for rigid-body using six parameters, derivative 12 parameters, voxel-specific 12 regressors (Chao-Gan et al., 2013). To further rule out the residual effect of motion on rsfMRI measures, volume-level mean framewise displacement was computed and used at the second-level analyses as covariates (Power et al., 2012).

There is a fierce recent controversy regarding whether the mean timecourse of whole brain should be regressed out in the rsfMRI analysis (Takeuchi et al., 2012). Regressing out the mean timecourse of whole brain may have merits (Chao-Gan et al., 2013) as well as weaknesses (Murphy et al., 2009). Considering this controversy, for fALFF, regional homogeneity and RSFC, we performed both analyses of images in which mean timecourse of whole brain is regressed out and analyses of images in which mean timecourse of whole brain is not regressed out. For DC, we did not regress out the mean timecourse of whole brain because it partly reflects global brain activity (Schölvinck et al., 2010), and conceptually, regressing out mean timecourse of whole brain is particularly problematic for calculating DC, which is the correlation between one voxel and the rest of the whole brain. The remaining procedures for group level multiple regression analyses were performed as described below.

The processed images were spatially smoothed with $7.5-\mathrm{mm}$ FWHM, and the resultant images were masked with the whole brain mask that was created as described above. The smoothing value was $7.5 \mathrm{~mm}$, because this value is twice the voxel size of the original image.

\section{Individual-level functional imaging data analysis for DC/fALFF/ regional homogeneity}

The descriptions of fALFF analyses and DC analyses were mostly reproduced from our previous study using identical methods (Takeuchi et al., 2015a). The fALFF analyses were performed using the DPARSF software as previously described similarly (Han et al., 2011; Wang et al., 2011). The time series of each voxel was transformed into the frequency domain, and the power spectrum was obtained. Because the power of a given frequency is proportional to the square of the amplitude of that frequency component, the square root was calculated at each frequency of the power spectrum, and the average square root was then obtained across $0.01-0.08 \mathrm{~Hz}$ at each voxel. This average square root was taken as ALFF. For fALFF, as described previously (Chao-Gan and Yu-Feng, 2010; Wang et al., 2012), a ratio of the power of each frequency at a low frequency range to that of the entire frequency range (fALFF) was computed. Specifically, after preprocessing, the time series for each voxel was transformed into the frequency domain without band-pass filtering. The square root was calculated at each frequency of the power spectrum. The sum of the amplitudes across $0.01-0.08 \mathrm{~Hz}$ was divided by that of the entire frequency range.

After preprocessing, the fMRI data were temporally band-pass filtered $(0.01<\mathrm{f}<0.08 \mathrm{~Hz})$ to reduce low frequency drift and high frequency. Furthermore, weighted DC measures were calculated using DPARSF, as previously described similarly (Wang et al., 2014). In brief, Pearson correlation coefficients were first computed between the time series of all pairs of gray matter voxels, leading to a whole-brain functional connectivity matrix for each individual. We calculated this in the areas of gray matter signal is $>.2$ in the aforementioned gray matter template, consistent with previous studies (Wang et al., 2014). Individual correlation matrices were transformed into a Z-score matrix using Fisher's r-to-z transformation to improve normality. We further computed weighted DC strength of a voxel as the sum of the connections (Z-values) between a given voxel and all other voxels. As has been performed elsewhere (Buckner et al., 2009), we conservatively restricted our analysis to positive correlations above a threshold of $\mathrm{r}=0.25$. Finally, standardized weighted DC maps were acquired by subtracting the mean value within the abovementioned gray matter mask, and then dividing by the standard deviation of whole gray matter mask (Yan et al., 2013). Voxels with higher DC values reflect the sum of the connections for a given voxel and all other voxels, and are thus indicative of their central roles in transferring information across brain regions. Z-standardization was performed only for DC measures. A previous study suggested the importance of application of z-standardization to rsfMRI measures to mitigate the association between motion and rsfMRI measures. However, such effects of z-standardization were not statistically clear in the case of fALFF when other methods to mitigate the effects of motion were also performed (see Supplimental Fig. S7 of Chao-Gan et al., 2013).

For the RSFC analysis, correlation maps were produced by extracting the BOLD time course from the seed regions and computing the correlation coefficient between this time course and the time course from all other brain voxels. In this study, we examined correlations of activity of the MPFC, which is a key node of the DMN, and the seed that generated significant results in our previous study (Takeuchi et al., 2012). The seed region of the $\mathrm{mPFC}$ was a sphere with a 6 -mm radius centered on the focus. The peak voxel of the $\operatorname{mPFC}(x, y, z=-1,47,-4)$ 
was defined as previously described (Fox et al., 2005; Takeuchi et al., 2012). Correlation values were Fisher z-transformed to improve normality. Finally, RSFC maps were acquired by subtracting the mean value within the whole-brain mask and dividing by the standard deviation of the whole-brain mask (Yan et al., 2013).

Subsequently, we calculated regional homogeneity images according to previously described procedures (Yao et al., 2009). Initially, spatially normalized (but not smoothed) rsfMRI images were bandpass filtered $(0.01<\mathrm{f}<0.08 \mathrm{~Hz})$ and masked using the whole brain mask. A regional homogeneity value was then calculated to measure similarity of the time series of a given voxel with its nearest 26 voxels. After normalization, the resulting images were spatially smoothed using 7.5-mm FWHM. Normalization of regional homogeneity maps was performed by dividing the regional homogeneity among each voxel by the average regional homogeneity of the whole brain. This regional homogeneity value is known as Kendall's coefficient concordance (KCC) (Kendall and Gibbons, 1990), and is used to measure the similarity of a time series of a given voxel with that of its nearest voxels in a voxel-wise way, based on the assumption that voxels are temporally similar to their neighbors (Tononi et al., 1998). Using this method, we measured temporal homogeneity of regional BOLD signals, which reflected the temporal homogeneity of neural activity. The formula to calculate $\mathrm{KCC}$ has been expounded in previous studies (Zang et al., 2004).

\section{Second-level statistical analysis}

Second-level statistical analyses were performed using SPM8 software. Areas included in these analyses were limited to those of the abovementioned gray matter mask. In whole brain analyses, we used voxel-wise analysis of covariance (ANCOVA), with sex difference as a grouping factor (using the full factorial option of SPM8). In this analysis, age, RAPM score, volume-level mean framewise displacement, as calculated according to a previous study (Power et al., 2012), and S-A creativity test scores were covariates. These covariates were modeled so that each had a unique relationship with rsfMRI measures for each sex except in the case of volume-level mean framewise displacement (using the interactions option in SPM8), which enabled investigation of the effects of interaction between sex and each covariate. In these analyses, the centering option was used for centering the interactions. The main effects of the S-A creativity test score (contrasts of [the effects of the S-A creativity test score for males, that for females] were [1 1] or [-1 -1]) and the interaction between sex and the S-A creativity test score (contrasts of [the effect of the S-A creativity test for males, the effect of the S-A creativity test for females] were $\left[\begin{array}{ll}-1 & 1\end{array}\right]$ or $\left.\left[\begin{array}{ll}1 & -1\end{array}\right]\right)$ were assessed using t-contrasts.

A multiple comparison correction of the cross-sectional analyses was performed using threshold-free cluster enhancement (TFCE) (Smith and Nichols, 2009), with randomized (5000 permutations) nonparametric permutation testing via the TFCE toolbox (http://dbm. neuro.uni-jena.de/tfce/). We applied a threshold of $P<0.05$ and corrected for multiple comparisons (FWE) based on the results of a permutation test using the TFCE value. Permutation-based correction for multiple comparisons is known to accurately control the false positive rate in real brain data (Silver et al., 2012).

For the RSFC analysis of the mPFC, if significant results were not
Table 1

Distribution of S-A creativity test scores for males and females in our sample.

\begin{tabular}{lllllllll}
\hline & $\mathrm{x} \leq 10$ & $10<$ & $20<$ & $30<$ & $40<$ & $50<$ & $60<$ & $70<\mathrm{x}$ \\
& & $\mathrm{x} \leq 20$ & $\mathrm{x} \leq 30$ & $\mathrm{x} \leq 40$ & $\mathrm{x} \leq 50$ & $\mathrm{x} \leq 60$ & $\mathrm{x} \leq 70$ & \\
Male & 2 & 34 & 153 & 272 & 183 & 72 & 15 & 1 \\
Female & 1 & 11 & 99 & 194 & 165 & 63 & 12 & 0 \\
\hline
\end{tabular}

obtained at the whole-brain level, region of interest (ROI) analysis was performed. This procedure was used to confirm the previously observed associations between the CMDT and RSFC and between the $\mathrm{mPFC}$ and posterior DMN. The correction for multiple comparisons was within an anatomical area that corresponded well to the posterior DMN. The ROI was constructed by adding the bilateral posterior and middle cingulate gyrus and the bilateral precuneus using the WFU PickAtlas Tool's aal option (http://www.fmri.wfubmc.edu/cms/ software\#PickAtlas).

\section{Results}

Basic data

Mean volume-wise framewise displacement calculated by the method of Power et al. (2012) was 0.165 (SD: 0.042, range: 0.092-0.413). The S-A creativity test score of males (mean: 37.56 , SD: 10.64, range: 7-71) was significantly smaller than that of females (mean: 39.34, SD: 9.96, range: 7-68) ( $\mathrm{P}=0.002, \mathrm{t}=3.07$, t-test). The distribution of S-A creativity test scores is presented in Table 1 . The demographic variables of age, RAPM scores, and S-A creativity test scores are presented in Table 2. The RAPM scores were slightly higher in males than in females ( 28.76 vs. 28.03 ); however, it is unlikely that these differences affected the significant findings from the rsfMRI analyses. The removal of subjects with the highest RAPM scores $(\geq 35)$ from the male sample (40 out of 732 subjects) and the lowest RAPM scores ( $\geq 20$ ) from the female sample (19 out of 545 subjects) equalized the RAPM scores (28.38 vs. 28.36) but did not alter significance of all significant rsfMRI findings identified in this study.

\section{Effects of the CMDT on $f A L F F$}

When the global signal was regressed out in individual analyses, ANCOVA revealed no significant overall negative or positive main effects (regardless of sex) of the S-A creativity test score on fALFF and no significant interaction effects between sex and S-A creativity test score on AALFF in any of the regions.

When the global signal was not regressed out in individual analyses, ANCOVA revealed no significant overall negative or positive main effects (regardless of sex) of the S-A creativity test score on fALFF. However, there was a significant effect of interaction between sex and S-A creativity test score (moderated by a negative correlation in males and a positive correlation in females) on fALFF in the areas of the middle cingulate cortex and precuneus, left middle temporal gyrus, cerebellum, and right middle frontal gyrus (Fig. 1; for statistical values, see Table 3). The simple correlation coefficient between mean fALFF in each cluster and the S-A Creativity test score is presented in Table 3. The interactions are mostly formed by both the tendency of negative

Table 2

Demographic variables and statistical comparisons between males and females.

\begin{tabular}{|c|c|c|c|c|c|c|c|c|c|c|c|c|}
\hline \multirow[t]{2}{*}{ Measure } & \multicolumn{5}{|l|}{ Males } & \multicolumn{5}{|c|}{ Females } & \multirow[t]{2}{*}{$P$ value } & \multirow[t]{2}{*}{$\mathrm{T}$ value } \\
\hline & Mean & SD & range & Kurtosis & skewness & Mean & $\mathrm{SD}$ & range & Kurtosis & skewness & & \\
\hline Age & 20.83 & 1.89 & $18-27$ & 0.770 & 0.258 & 20.67 & 1.62 & $18-27$ & 0.638 & 0.823 & 0.110 & -1.60 \\
\hline RAPM & 28.76 & 3.86 & $13-36$ & -0.516 & 0.289 & 28.03 & 3.83 & $15-36$ & -0.371 & -0.024 & $<0.001$ & -3.33 \\
\hline S-A creativity test & 37.55 & 10.64 & $7-71$ & 0.234 & 0.073 & 39.34 & 9.96 & $7-68$ & 0.182 & -0.039 & 0.002 & 3.08 \\
\hline
\end{tabular}



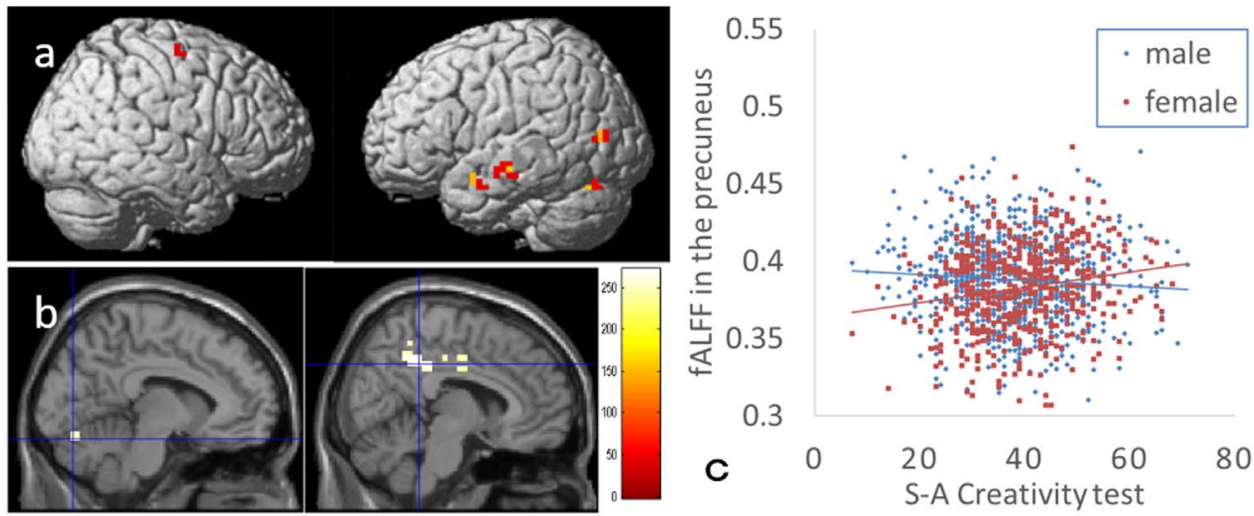

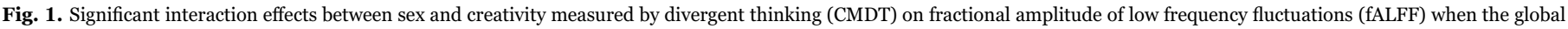

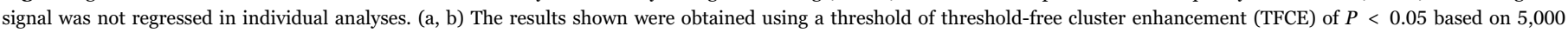

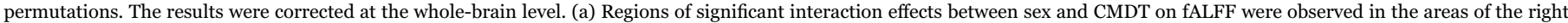

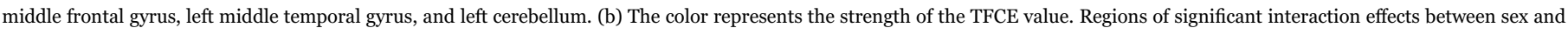

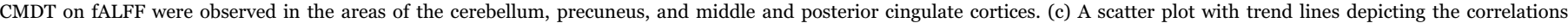

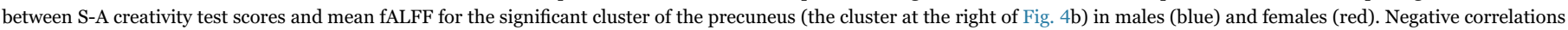
in males and positive correlations in females were observed.

correlation in males and that of positive correlations in females. However, in case of one cluster in the cerebellum, the positive correlations in females are low. On the other hand, in the case of two clusters in the precuneus and the middle cingulate gyrus, the negative correlations in males are low. Post-hoc multiple regression analyses of data from females only did not show any significant correlations between S-A creativity test score and fALFF. However, post-hoc multiple regression analyses of data from males only showed significant negative correlations between S-A creativity test score and fALFF in the cerebellum and the contingent areas (Supplemental Fig. 8).

\section{Effects of the CMDT on DC}

ANCOVA revealed no significant overall negative or positive main effects (regardless of sex) of the S-A creativity test score on DC, and no significant interaction effects between sex and S-A creativity test score on DC in any of the regions.

\section{Effects of the CMDT on regional homogeneity}

When the global signal was regressed out in individual analyses, ANCOVA revealed no significant overall negative or positive main effects (regardless of sex) of the S-A creativity test score on regional homogeneity. However, there were significant interaction effects between sex and S-A creativity test score on regional homogeneity in the anatomical area that included the anterior part of the middle temporal gyrus and left temporal pole $(x, y, z=-56.25,-3.75,-22.50, t=4.98$, TFCE value $=299.31, P=0.004$, corrected for multiple comparisons using TFCE, $3111 \mathrm{~mm}^{3}$, Fig. 2a). The simple correlation coefficient between mean regional homogeneity in this cluster and the S-A Creativity test score was $\mathrm{r}=-0.127$ for males $(\mathrm{p}=0.00059, \mathrm{t}=-3.452)$ and $\mathrm{r}=0.188$ for females $(\mathrm{p}=0.0000097, \mathrm{t}=4.465)$. Post-hoc multiple regression analyses of data from males only and from females only did not show any significant correlations between S-A creativity test score and regional homogeneity.

When the global signal was not regressed out in individual analyses, ANCOVA revealed no significant overall negative or positive main effects (regardless of sex) of the S-A creativity test score on regional homogeneity. However, there were significant interaction effects between sex and the S-A creativity test score on regional homogeneity in the anatomical area that included the anterior part of the middle temporal gyrus and left temporal pole ( $\mathrm{x}, \mathrm{y}, \mathrm{z}=-56.25,-3.75,-18.75$, $\mathrm{t}=4.95$, $\mathrm{TFCE}$ value $=330.59, \mathrm{P}=0.004$, corrected for multiple compar- isons using TFCE, $4482 \mathrm{~mm}^{3}$, Fig. 2b). The simple correlation coefficient between mean regional homogeneity in this cluster and the S-A Creativity test score was $r=-0.127$ for males $(p=0.00056, t=-3.468)$ and $\mathrm{r}=0.188$ for females $(\mathrm{p}=0.00002, \mathrm{t}=4.3084)$. Post-hoc whole-brain multiple regression analyses using data from females only (corrected for age, RAPM score, and volume-level mean frame-wise displacement) revealed a near-significant positive correlation between regional homogeneity and S-A creativity test score $(\mathrm{P}=0.070$, corrected for multiple comparisons using TFCE) in the left anterior temporal pole. In contrast, no such tendency was found in analysis of data from males.

Both of these significant interaction effects appear to be moderated by the negative correlation in males and positive correlation in females (Fig. 2ab).

Although, females showed slightly higher scores on the S-A creativity test, this was not the cause of the aforementioned interactions, as seen in the scatter plots of figures.

\section{Effects of CMDT on the RSFC with the MPFC}

When the global signal was regressed out in individual analyses, ANCOVAs revealed no significant overall main effects (regardless of sex) of the S-A creativity test score on RSFC with the mPFC or significant interactions between the S-A creativity test score and sex.

When the global signal was not regressed out in individual analyses, ANCOVAs revealed no significant overall main effects (regardless of sex) of the S-A creativity test score on RSFC with the MPFC at the whole-brain level. However, the ROI analysis within the posterior DMN (precuneus and middle and posterior cingulate cortices) revealed a significant overall positive main effect (regardless of sex) of the S-A creativity test score on the RSFC between the MPFC (the seed area) and the area around the precuneus and middle cingulate cortex $(\mathrm{x}, \mathrm{y}$, $\mathrm{z}=11.25,-33.75,37.50, \mathrm{t}=3.87$, TFCE value=117.62, $\mathrm{P}=0.022$, corrected for multiple comparisons using TFCE within the ROI, significant areas $=1160 \mathrm{~mm}^{3}$, Fig. 3ab). This finding is in accordance with our previous finding that showed the positive association between the CMDT and RSFC between the MPFC and posterior DMN; thus, this result is not discussed again in this study as this was topic of our previous study and the discussion was extensively done in our previous study and this analysis was done to confirm our previous findings (Takeuchi et al., 2012). Notably, the analysis in our previous study was performed with 160 subjects also included in our present study. In addition, ANCOVA revealed significant interaction effects between sex and the S-A creativity test score on the RSFC between the mPFC (the 
Table 3

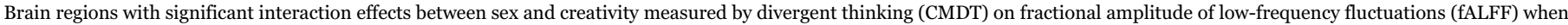
the global signal was not regressed in individual analyses.

\begin{tabular}{|c|c|c|c|c|c|c|c|c|c|}
\hline \multirow[t]{2}{*}{ Area } & & \multicolumn{3}{|c|}{ MNI coordinates } & \multirow[t]{2}{*}{ TFCE value } & \multirow[t]{2}{*}{ Corrected $P$ valuea ${ }^{\text {a }}$} & \multirow{2}{*}{$\begin{array}{l}\text { Cluster size } \\
\left(\mathrm{mm}^{3}\right)\end{array}$} & \multirow[t]{2}{*}{ Male correlation (r, t, p) } & \multirow[t]{2}{*}{ Female correlation $(\mathrm{r}, \mathrm{t}, \mathrm{p})$} \\
\hline & & $\mathrm{x}$ & $\mathrm{y}$ & $\mathrm{z}$ & & & & & \\
\hline $\begin{array}{l}\text { Middle cingulate cortex/ } \\
\text { Precuneus }\end{array}$ & & 7.5 & -41.25 & 37.5 & 271.22 & 0.032 & 2215 & $-0.074,-2.007,0.045$ & $0.179,4.251,2.5^{*} 10^{-5}$ \\
\hline Middle cingulate cortex & & 3.75 & -11.25 & 37.5 & 261.44 & 0.036 & 686 & $-0.096,-2.595,0.010$ & $0.115,2.705,0.007$ \\
\hline Middle temporal gyrus & $\mathrm{L}$ & -52.5 & -15 & -11.25 & 257.66 & 0.038 & 527 & $-0.113,-3.078,0.002$ & $0.104,2.445,0.015$ \\
\hline Cerebellum & $\mathrm{L}$ & -52.5 & -71.25 & -22.5 & 253.42 & 0.04 & 369 & $\begin{array}{l}-0.135,-3.678 \\
2.53^{*} 10^{-4}\end{array}$ & $0.101,2.355,0.019$ \\
\hline Cerebellum & $\mathrm{L}$ & -7.5 & -78.75 & -18.75 & 246.56 & 0.045 & 316 & $-0.151,-4.119,4.2^{*} 10^{-5}$ & $0.060,1.393,0.164$ \\
\hline Precuneus & $\mathrm{R}$ & 11.25 & -48.75 & 52.5 & 245.32 & 0.046 & 105 & $-0.052,-1.399,0.162$ & $0.113,2.646,0.008$ \\
\hline Middle cingulate cortex & $\mathrm{R}$ & 11.25 & -22.5 & 41.25 & 243.17 & 0.047 & 105 & $-0.016,-0.437,0.663$ & $0.149,3.521,4.66^{*} 10^{-4}$ \\
\hline Middle temporal gyrus & $\mathrm{L}$ & -52.5 & -78.75 & 7.5 & 243.05 & 0.047 & 369 & $-0.102,-2.781,0.006$ & $0.095,2.220,0.027$ \\
\hline Middle temporal gyrus & $\mathrm{L}$ & -60 & 7.5 & -18.75 & 242.38 & 0.047 & 422 & $-0.090,-2.444,0.015$ & $0.106,2.477,0.014$ \\
\hline Middle frontal gyrus & $\mathrm{R}$ & 30 & -7.5 & 60 & 240.24 & 0.048 & 158 & $-0.118,-3.198,0.001$ & $0.075,1.756,0.080$ \\
\hline
\end{tabular}

${ }^{\text {a }}$ Corrected for multiple comparison using the permutation test of threshold free cluster enhancement (TFCE) value.
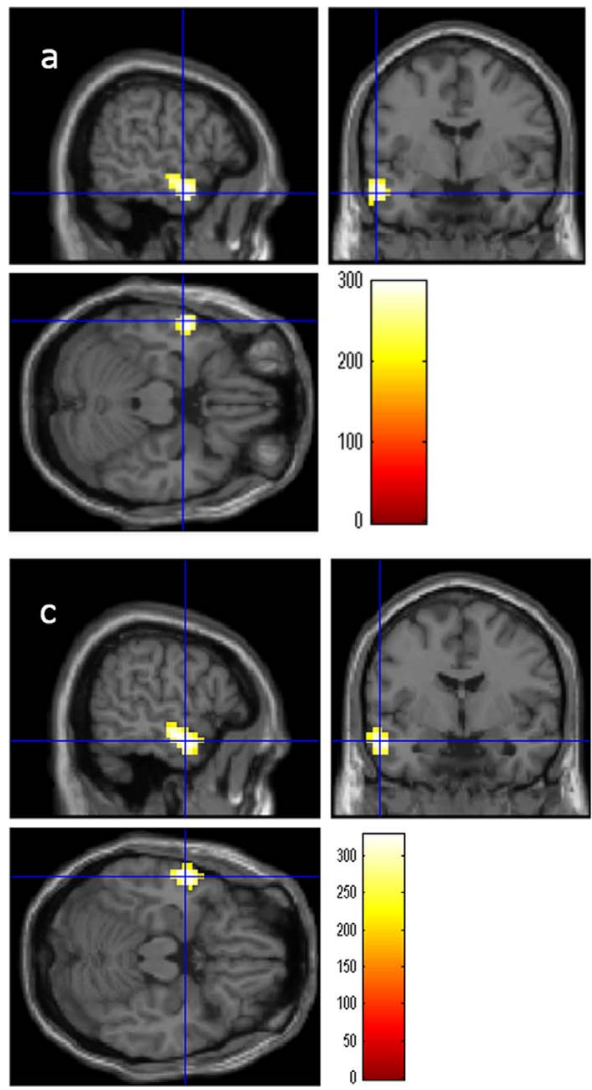

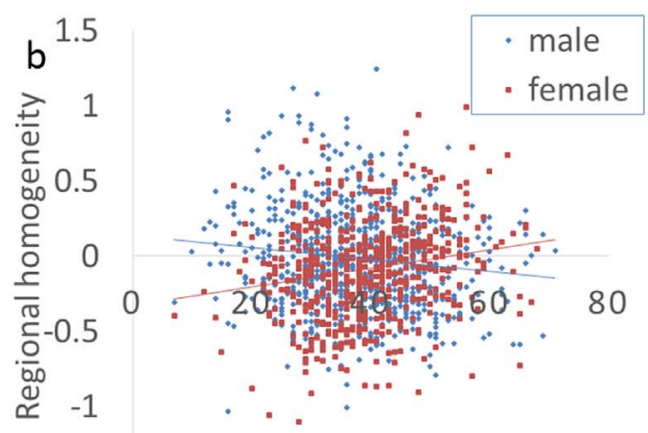

$-1.5$

\section{S-A Creativity test}

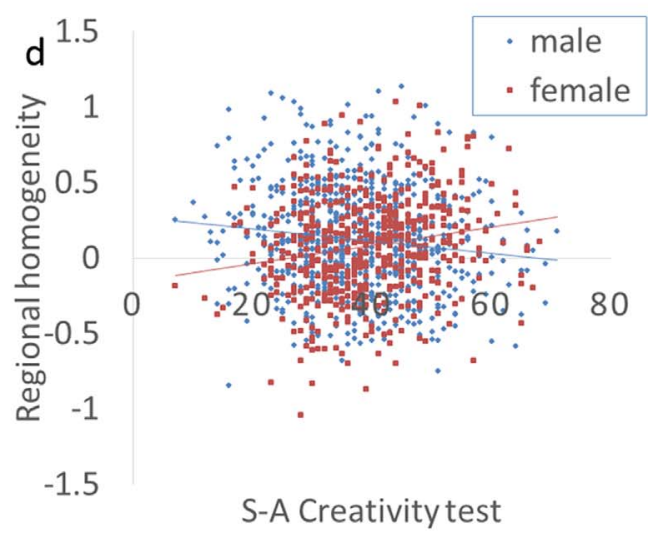

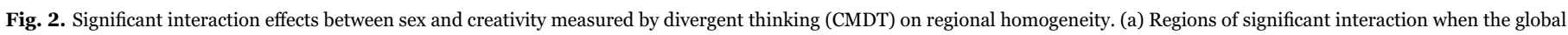

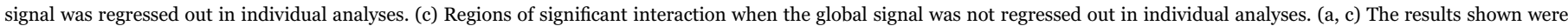

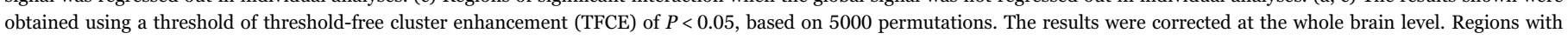

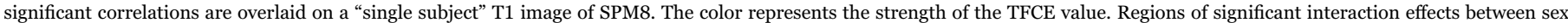

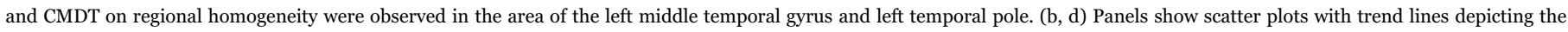

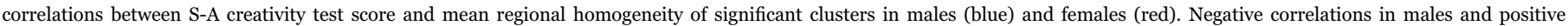

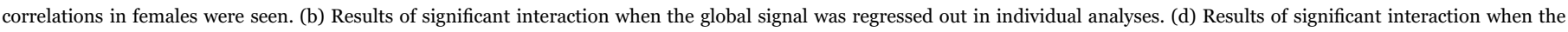
global signal was not regressed out in individual analyses.

seed area) and left inferior frontal gyrus ( $x, y, z=-41.25,22.50,3.75$, $\mathrm{t}=4.03$, $\mathrm{TFCE}$ value $=240.56, \mathrm{P}=0.048$, corrected for multiple comparisons using TFCE, $158 \mathrm{~mm}^{3}$, Fig. 4ab).

The simple correlation coefficient between the mean RSFC with the $\mathrm{mPFC}$ in this cluster and the S-A Creativity test score was $\mathrm{r}=-0.081$ for males $(\mathrm{p}=0.0281, \mathrm{t}=-2.200)$ and $\mathrm{r}=0.147$ for females $(\mathrm{p}=0.00057$, $\mathrm{t}=3.464$ ). Post-hoc whole-brain multiple regression analyses using data from females only (corrected for age, RAPM score, and volume-level mean frame-wise displacement) revealed a near-significant positive correlation between the RSFC with the $\mathrm{MPFC}$ and S-A creativity test score $(x, y, z=-37.5,7.5,0$, TFCE value $=232.27, \mathrm{P}=0.055$, corrected for multiple comparisons using TFCE) in the contingent area. The same analysis using data from males revealed a near-significant positive correlation between the RSFC with the $\mathrm{MPFC}$ and S-A creativity test 

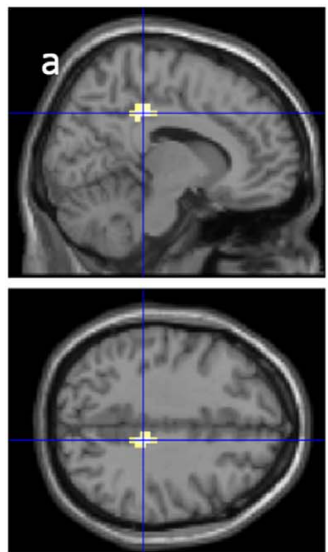

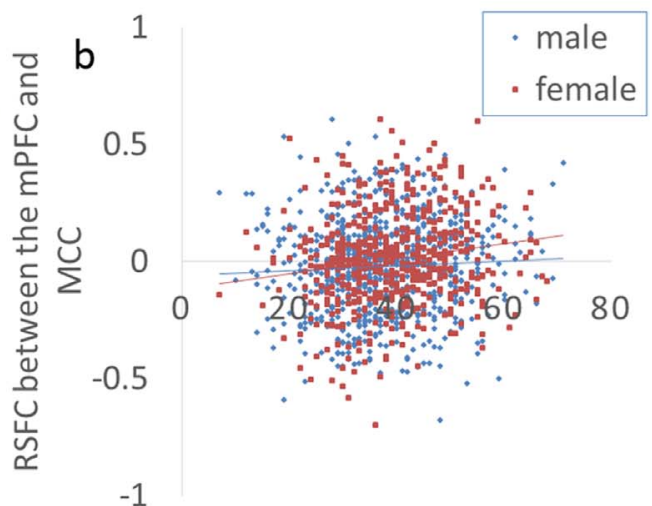

S-A Creativity test

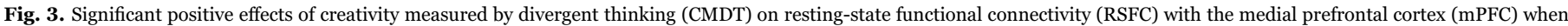

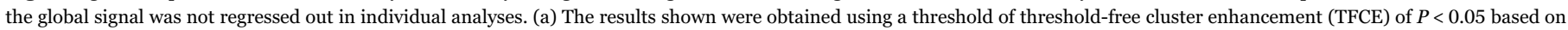

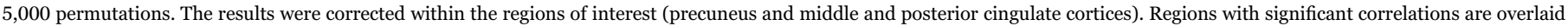

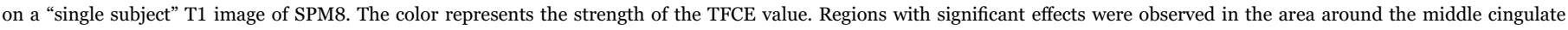

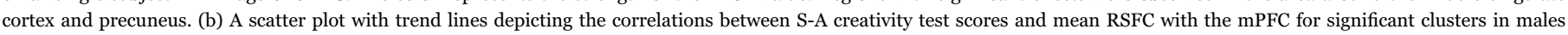
(blue) and females (red). Negative correlations in males and positive correlations in females were observed.
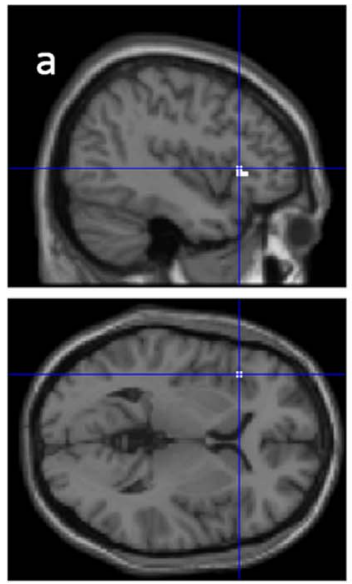

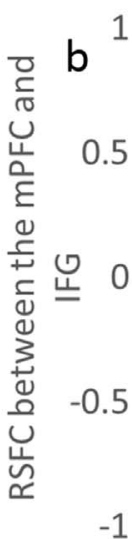

$-1$

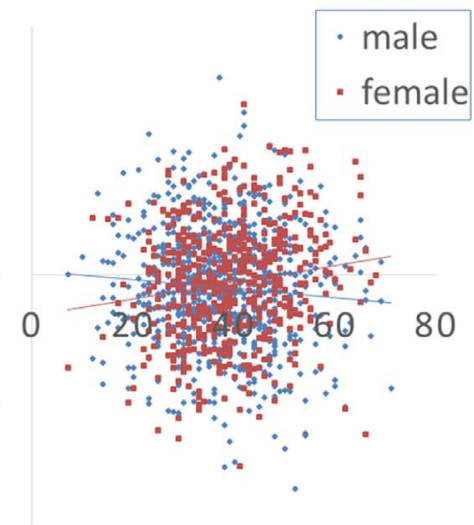

S-A Creativity test

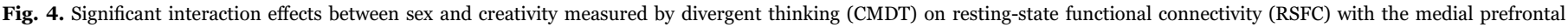

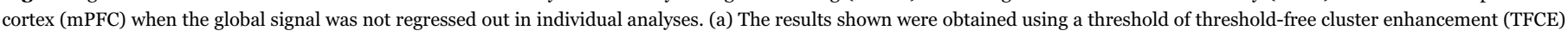

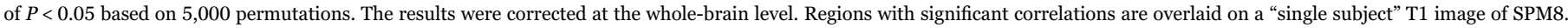

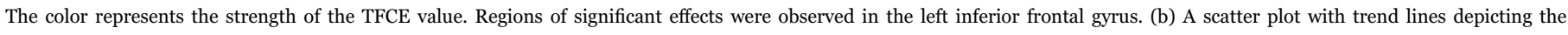

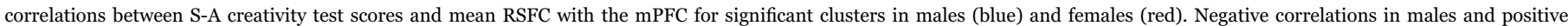
correlations in females were observed.

score $(x, y, z=-41.25,26.25,-11.25$, TFCE value $=232.28, P=0.067$, corrected for multiple comparisons using TFCE) in the contingent area.

The significant interaction effect appears to be mediated by a negative correlation in males and positive correlation in females (Fig. 4a). There were no significant interaction effects between sex and the S-A creativity test score on RSFC with the MPFC (the seed area) when ROI analysis was performed within the posterior DMN (precuneus and middle and posterior cingulate cortices), regardless if the global signal was regressed out in individual analyses.

\section{Discussion}

We investigated the association between fALFF/DC/regional homogeneity/RSFC with the MPFC and verbal CMDT as well as the presence of any sex differences. Our hypotheses were largely not supported as DC were not significantly associated with verbal CMDT. Partially consistent with our hypothesis, however, the whole brain analysis revealed a significant interaction between verbal CMDT and sex on regional homogeneity within an area from the left middle temporal gyrus to the left temporal pole (left anterior temporal lobe). This interaction was moderated by the relatively stronger positive correla- tions in females compared to the weak negative correlations in males, suggesting the importance of local connectivity of synchronization for CMDT specifically in females. These results are compelling in terms of the statistical significance. In addition, there was a significant interaction between verbal CMDT and sex on the RSFC between the mPFC and left inferior frontal gyrus, an effect that was mediated by a positive correlation in females and negative correlation in males. Similarly, there were significant interactions between verbal CMDT and sex on fALFF (when the global signal was not regressed in individual analyses) in several distinct areas, including the precuneus and middle cingulate gyrus, left middle temporal gyrus, right middle frontal gyrus, and cerebellum. These interactions were mostly moderated by both the tendency of positive correlations in females and negative correlation in males as seen in Table 3. Females had a slightly higher CMDT score. However, this difference does not explain the observed sex differences. This is because in this study we investigated sex differences in the correlations between the CMDT score and imaging measures. And in such investigation, a higher score in one group did not lead to group differences in correlations unless there is a non-linear association between the CMDT score and imaging measures in the whole population (including men and women). Further, mostly, the scores of males 
and females mostly overlapped as seen in Fig. 2; thus, even the existence of non-linear associations was unlikely to cause the observed sex differences in correlation patterns.

Generally, it is difficult to construe the findings of regional homogeneity. One of the most consistent findings regarding the associations between regional homogeneity and cognitive integrity is that regional homogeneity in the precuneus is reduced with the progression of Alzheimer's disease (Liu et al., 2008). Thus, regional homogeneity in the precuneus may reflect the integrity of overall cognitive function (Zhang et al., 2015). For other higher order cognitive function, although many previous findings are conflicting, the majority tend to suggest the better higher order cognitive functions are related to greater regional homogeneity of the areas that play critical roles in the relevant higher order cognitive functions (Jiang and Zuo, 2015). Thus, coordinated work (which may underlie stronger regional homogeneity) of the areas that play a crucial role in the cognition may contribute to the better functions. However, the cellular mechanisms underlying individual differences in resting-state regional homogeneity are still largely unknown (Jiang and Zuo, 2015) and future studies are required to investigate this issue.

Higher regional homogeneity in the anterior temporal lobe may facilitate the effective development of ideas, particularly creative ideas through combination of disparate information. The anterior temporal lobe is thought to serve as a semantic hub linking conceptual information distributed throughout the brain and to represent such combinatorial concepts (for review, see Simmons and Martin, 2009). It is also suggested to contribute to the computation of combinatorial meaning (Hickok and Poeppel, 2007) and to be important for judging the conceptual similarity of different information (Rogers et al., 2006). On the other hand, according to James (1890), creativity requires "transitions from one idea to another...unheard of combination of elements, the subtlest associations of analogy...where partnerships can be joined or loosened...". Heilman et al. (2003) also stated a possible method of resolving a previously unsolved problem is to see the problem "in a new light". A means of viewing the problem in a new light is to use a different form of knowledge. Considering these, one intriguing speculation is that stronger functional connectivity among areas (regional homogeneity) that play a key role in representing concepts and combining different concepts may facilitate the generation of ideas, particularly creative ones, by allowing novel combinations of disparate information.

We suggest that the present results are congruent with this idea that the combination of disparate ideas is important for creativity in females specifically. The combination of ideas has traditionally been thought important for creativity, so by extension, brain connectivity is important for creativity. However, our recent study using a large sample showed the CMDT was positively associated with regional white matter volume only in females (Takeuchi et al., in press). Based on our previous and present studies, which both used a large sample, we suggest that certain types of idea combination may be important for creativity specifically in females, at least in the case of CMDT. To completely substantiate this idea, psychological experiments are necessary in which the associations between CMDT performance and different tasks requiring the combination of disparate information (such as the remote associate task) are investigated separately in males and in females.

Interpretation of the negative correlation between regional homogeneity in the left anterior temporal lobe and CMDT in males requires caution because (a) the strength of these associations in males were substantially weaker than the positive associations in females and (b) when the whole brain signal was not regressed out in individual analyses, whole brain analyses of data from males showed no tendencies for negative correlations between CMDT and regional homogeneity in the left anterior temporal lobe, while there was a near significant tendency for a positive correlation when the data from females alone was analyzed. As discussed by Vul et al. (2009), significant findings in whole brain analyses suffer from overfitting to the contrast. Here this means that even when there are only positive associations in females leading to significant interaction effects between sex and regional homogeneity, the significant voxels of this interaction effect tend to show a negative correlation in males as well even in the absence of true effects. Regardless, the negative correlation between CMDT and regional homogeneity in males was far from significant in terms of the whole-brain correction for multiple comparisons, and the associations were relatively weak (lower $r$ values). Future studies are needed to ascertain these negative associations in males. However, a nearsignificant negative association of CMDT and the RSFC between the mPFC and IFG was observed in males. Thus, the negative connectivity patterns may be more robust. These negative correlations may be reflective of the fact that creativity was more strongly associated with psychopathology in males (Martín-Brufau and Corbalán, 2016), which is associated with reduced functional connectivity (Broyd et al., 2009).

On the other hand, in females, there was a stronger positive association of verbal CMDT with the RSFC between the MPFC and IFG, which have important roles in default state cognitive activity (Buckner et al., 2008) and internal speech, respectively (Aziz-Zadeh et al., 2005). In addition, IFG lesions led to a deficit in speech production, which in turn lead to reduction in creativity (Flaherty, 2005). Furthermore, it has been suggested that default-state cognitive activity is important for creativity (Takeuchi et al., 2012). Thus, the efficient functional connection between the MPFC and left IFG may be important for generating verbal production in the default cognitive state and lead to a greater CMDT. Another interpretation of a greater RSFC between the mPFC and left IFG is the increased interaction between areas that belong to different networks. It has been reported that the RSFC of areas belonging to different functional networks increases during divergent thinking (Beaty et al., 2015). The mPFC belongs to the DMN and the left IFG belongs to a network activated during an externally directed attention demanding task (Fox et al., 2005); thus, the increased RSFC between these regions can be interpreted as an increased RSFC between areas of different networks. The increased interaction between areas that process different types of information, may lead to increased creativity, as combination of different types of information lead to creativity (Heilman et al., 2003). This type of information processing may lead to increased CMDT in females with greater RSFC between the mPFC and left IFG. However, these are speculations based on reverse inferences and need to be confirmed in future studies using other experimental methods. On the other hand, a previous study has shown that mPFC lesions and lesions affecting both the mPFC and (left or right) IFG led to a significant reduction in CMDT, whereas lesions of the (left or right) IFG alone did not significantly reduce CMDT (Shamay-Tsoory et al., 2011). Furthermore, transcranial direct current stimulation (tCDS) studies have shown that inhibitory stimulation of the left IFG facilitated the generation of creative responses (Chrysikou et al., 2013) as well as CMDT (Mayseless and Shamay-Tsoory, 2015). A study on artistic development in patients with frontotemporal dementia suggested that language is not required for, and may even inhibit, certain types of visual creativity (Mell et al., 2003). Therefore, the association between CMDT and the RSFC between the MPFC and left IFG may be more complex than the abovementioned interpretation. The femalespecific positive correlation between rWMV and CMDT observed in our other study (Takeuchi et al., in press) and the positive correlation between regional homogeneity and CMDT in the left temporal pole observed in this study generally support the idea that greater brain connectivity is important in females. However, these findings appear to contradict the results of the work done by Ryman et al. (2014). These authors utilized DTI and performed analyses of new metrics of connectivity organization, including connectivity strength, clustering, and communication efficiency, using data from 83 subjects. Their results suggest the existence of female-specific negative associations between these measures and CMDT. Little is known about these 
measures, and the association between these measures and other connectivity measures, such as rsfMRI and rWMV, as well as their implications should be investigated in future studies.

A previous study has shown the association between a positive correlation of CMDT and the RSFC between the MPFC and left IFG in a relatively small sample (including males and females) (Beaty et al., 2014). In comparison with this previous study, our study is congruent with the findings in females but not in males. This discrepancy may be due to the use of different methods (e.g., sample and CMDT measures) or sample sizes. Regardless, future studies are needed to address these issues.

As for the results of fALFF, the significant interactions between verbal CMDT and sex on AALFF that were moderated by the contrasts of negative associations in males and positive associations in females were found in several areas. fALFF, is suggested to indicate magnitudes of spontaneous neural activity during rest (Yan and Zang, 2010; Yang et al., 2015; Zang et al., 2007). Consistently, repetition rates of visual stimuli induce changes in fALFF just like it does to BOLD signals to the visual cortex ( $\mathrm{Li}$ et al., 2011). Furthermore, regional metabolic rates of the brain are strongly positively associated with fALFF (Tomasi et al., 2013). Thus, the observed interaction suggests that females tend to show positive associations between magnitudes of spontaneous neural activity during rest and CMDT, whereas males show the opposite pattern. These patterns of negative correlation in males and positive correlation in females were seen in the case of other rsfMRI measures as well as white matter volume measures (Takeuchi et al., in press). Although CMDT shows positive correlations with other cognitive functions such as processing speed in males and females (Hong et al., 2015; Preckel et al., 2011), a psychological study reported that the association between creativity and psychopathology differs between the sexes, and a stronger positive association is seen among males (Martín-Brufau and Corbalán, 2016). In addition, psychopathology often negatively correlates with fALFF (Sato et al., 2016), RSFC measures (Broyd et al., 2009), and brain volume measures (Hong et al., 2015). Perhaps, owing to these positive associations between psychopathology and CMDT in males, male associations between CMDT and brain indices may become more complex.

The areas of significant interaction on fALFF include precuneus/ middle cingulate gyrus, left middle temporal gyrus, right middle frontal gyrus, and cerebellum. As for the precuneus, the correlations were positive in females and males showed little correlations in this area. We previously reported that higher CMDT is associated with reduced taskinduced deactivation in the precuneus (Takeuchi et al., 2011c), lower regional cerebral blood flow in the precuneus (Takeuchi et al., 2011a), and higher resting-state functional connectivity between the precuneus and mPFC (Takeuchi et al., 2012). As previously described, the precuneus is an important node in the DMN where the information from the subsystems of the DMN is thought to converge (Buckner et al., 2008). The precuneus is supposed to play an important role in cognitive processes that are ongoing during rest and when engaged in cognitive activities such as self-related mental representations and episodic memory retrieval (Cavanna and Trimble, 2006). Perhaps these cognitive processes during rest contribute to CMDT in females. One of the areas of significant effects between sex and verbal CMDT included areas in the areas of the left middle temporal lobe. The areas in the left middle temporal lobe are associated with language. A previous study showed that verbal CMDT is associated with greater RSFC between the mPFC and left middle temporal gyrus (Wei et al., 2013). The anterior parts' functions were as described above, but the posterior middle temporal lobe is involved in functions such as semantic decisions (Whitney et al., 2011). The cerebellum has numerous functions; thus, it is difficult to estimate the functions that are relevant in this context; however, one of the major functions of this area is the phonological loop function (Baddeley, 2003). The phonological loop is assumed to possess verbal and acoustic information using a temporary store and an articulatory rehearsal system (Baddeley, 2003). Thus, one possibility is that the associations between the fALFF and verbal CMDT in these may be that in females these language-related functions are important for the effective production of ideas in verbal divergent thinking; in contrast, in males, the opposite occurs. In addition, the areas of significant interaction on fALFF included the areas where the effects of significant interaction on regional homogeneity were observed. Therefore, the observed findings of fALFF and those of homogeneity may be related to each other and may contribute to each other [greater activity may lead to greater local connectivity measure and greater local connectivity may lead to greater activity]. Finally, the right middle frontal gyrus is involved in generating unusual or distant verbal associations, both of which apparently characterize creative thinking (Kiefer et al., 1998; Seger et al., 2000). Finally, the functional activation study showed that greater CMDT is associated with greater activity in the areas of the bilateral middle frontal lobes during divergent thinking (Chávez-Eakle et al., 2007). In addition, musicians and schizotypes, which are both characterized by greater CMDT, show greater activity in the right prefrontal cortex and the bilateral middle frontal lobe, respectively (Folley and Park, 2005; Gibson et al., 2009). Therefore, the observed interaction in this area may suggest that the default activity of this area as well as the corresponding functions of this area (generating unusual or distant verbal associations) differently contribute to CMDT in males and females. However, the discussions in this paragraph depend on speculations based on the reverse inference and cannot be concluded from the present study. In addition, the significance levels of these results of fALFF were $0.032<\mathrm{P}<0.05$, and they were not supported by our hypothesis or previous studies' relevant findings unlike the cases of significant results of other analyses. Thus, given the number of analyses and contrasts, the results of fALFF may be obtained with caution.

This study had a few limitations that are common to many previous studies of creativity. First, as discussed in our previous studies (Takeuchi et al., 2011b), our sample consists of young, highly educated, healthy subjects, a common sample type because many studies use college cohorts (Jung et al., 2010; Song et al., 2008; Takeuchi et al., 2011b). However, this is also a strength because among subjects of below-normal intelligence, general intelligence is suggested to be positively correlated with creativity (Sternberg, 2005). Thus, focusing on a sample population with a relatively high level of education is important to disentangle the complex association between intelligence and CMDT. Further, we did not gather information on the menstrual cycles of females. It has been previously reported that the menstrual cycle was associated with alterations in the RSFC between the mPFC and angular gyrus; dorsolateral prefrontal cortex and sensorimotor cortex; dorsolateral prefrontal cortex and hippocampus; and dorsolateral prefrontal cortex and anterior cingulate gyrus (Hjelmervik et al., 2014; Petersen et al., 2014). These RSFCs are irrelevant to the significant findings in our study. However, changes in the menstrual cycle led to changes in CMDT (Krug et al., 1994); thus, unknown associations between rsfMRI measures and menstrual cycles can theoretically lead to the formation of associations between rsfMRI measures and CMDT in females. Future studies should investigate if the present findings can be explained by menstrual cycle differences.

In conclusion, there were significant interaction effects of CMDT and sex on (a) regional homogeneity in the anterior left temporal lobe, (b) the RSFC between the MPFC (the seed area) and left IFG and (c) fALFF in the areas of the right middle frontal lobe, precuneus, left middle temporal gyrus, and cerebellum. The interaction appears to be moderated, at least in part, by a positive correlation between CMDT and these measures in females. These findings suggest that greater CMDT in females is reflected by (a) regional coherence of brain areas representing and combining concepts (b) the efficient functional connection between the areas that play key roles in default-state cognitive activity and speech production and (c) greater spontaneous neural activity during the resting of brain areas involved in frontal lobe functions, default cognitive activities, and language functions. Further, 
these findings suggest that associations between creativity and resting state brain connectivity patterns are different between males and females.

\section{Acknowledgments}

We thank Yuki Yamada for operating the MRI scanner, Haruka Nouchi for conducting the psychological tests, all other assistants for helping with the experiments and the study, the study participants, and all our other colleagues at IDAC, Tohoku University for their support. This study was supported by JST/RISTEX, JST/CREST, a Grant-in-Aid for Young Scientists (B) (KAKENHI 23700306), and a Grant-in-Aid for Young Scientists (A) (KAKENHI 25700012) from the Ministry of Education, Culture, Sports, Science, and Technology.

\section{Appendix A. Supplementary material}

Supplementary data associated with this article can be found in the online version at doi:10.1016/j.neuroimage.2017.02.079.

\section{References}

Abraham, A., Thybusch, K., Pieritz, K., Hermann, C., 2014. Gender differences in creative thinking: behavioral and fMRI findings. Brain Imaging Behav. 8, 39-51.

Aziz-Zadeh, L., Cattaneo, L., Rochat, M., Rizzolatti, G., 2005. Covert speech arrest induced by rTMS over both motor and nonmotor left hemisphere frontal sites. J. Cogn. Neurosci. 17, 928-938.

Baddeley, A., 2003. Working memory: looking back and looking forward. Nat. Rev.: Neurosci. 4, 829-839.

Baer, J., Kaufman, J.C., 2008. Gender differences in creativity. J. Creat. Behav. 42, $75-105$.

Beaty, R.E., Benedek, M., Kaufman, S.B., Silvia, P.J., 2015. Default and executive network coupling supports creative idea production. Sci. Rep., 5.

Beaty, R.E., Benedek, M., Wilkins, R.W., Jauk, E., Fink, A., Silvia, P.J., Hodges, D.A., Koschutnig, K., Neubauer, A.C., 2014. Creativity and the default network: a functional connectivity analysis of the creative brain at rest. Neuropsychologia 64, 92-98.

Broyd, S.J., Demanuele, C., Debener, S., Helps, S.K., James, C.J., Sonuga-Barke, E.J.S., 2009. Default-mode brain dysfunction in mental disorders: a systematic review. Neurosci. Biobehav. Rev. 33, 279-296.

Buckner, R.L., Andrews-Hanna, J.R., Schacter, D.L., 2008. The brain's default network. Ann. N. Y. Acad. Sci. 1124, 1-38.

Buckner, R.L., Sepulcre, J., Talukdar, T., Krienen, F.M., Liu, H., Hedden, T., AndrewsHanna, J.R., Sperling, R.A., Johnson, K.A., 2009. Cortical hubs revealed by intrinsic functional connectivity: mapping, assessment of stability, and relation to Alzheimer's disease. J. Neurosci. 29, 1860-1873.

Cavanna, A.E., Trimble, M.R., 2006. The precuneus: a review of its functional anatomy and behavioural correlates. Brain 129, 564-583.

Chao-Gan, Y., Brian, C., Clare, K., Stan, C., R Cameron, C., Adriana Di, M., Qingyang, L., Xi-Nian, Z., F Xavier, C., Michael P, M., 2013. A comprehensive assessment of regional variation in the impact of head micromovements on functional connectomics. Neuroimage 76, 183-201.

Chao-Gan, Y., Yu-Feng, Z., 2010. DPARSF: a MATLAB toolbox for "pipeline" data analysis of resting-state fMRI. Front. Syst. Neurosci. 13, 11-17, (4, article).

Chávez-Eakle, R.A., Graff-Guerrero, A., García-Reyna, J.C., Vaugier, V., Cruz-Fuentes, C., 2007. Cerebral blood flow associated with creative performance: a comparative study. Neuroimage 38, 519-528.

Chen, Q.-L., Xu, T., Yang, W.-J., Li, Y.-D., Sun, J.-Z., Wang, K.-C., Beaty, R.E., Zhang, Q.L., Zuo, X.-N., Qiu, J., 2015. Individual differences in verbal creative thinking are reflected in the precuneus. Neuropsychologia 75, 441-449.

Chrysikou, E.G., Hamilton, R.H., Coslett, H.B., Datta, A., Bikson, M., Thompson-Schill, S.L., 2013. Noninvasive transcranial direct current stimulation over the left prefrontal cortex facilitates cognitive flexibility in tool use. Cogn. Neurosci. 4, 81-89.

Cole, M.W., Yarkoni, T., Repovš, G., Anticevic, A., Braver, T.S., 2012. Global connectivity of prefrontal cortex predicts cognitive control and intelligence. J. Neurosci. 32, 8988-8999.

Damoiseaux, J.S., Rombouts, S., Barkhof, F., Scheltens, P., Stam, C.J., Smith, S.M., Beckmann, C.F., 2006. Consistent resting-state networks across healthy subjects. In: Proceedings of the National Academy of Sciences. vol. 103, pp.13848-13853.

Flaherty, A.W., 2005. Frontotemporal and dopaminergic control of idea generation and creative drive. J. Comp. Neurol. 493, 147-153.

Folley, B.S., Park, S., 2005. Verbal creativity and schizotypal personality in relation to prefrontal hemispheric laterality: a behavioral and near-infrared optical imaging study. Schizophr. Res. 80, 271-282.

Fox, M.D., Snyder, A.Z., Vincent, J.L., Corbetta, M., Van Essen, D.C., Raichle, M.E., 2005. The human brain is intrinsically organized into dynamic, anticorrelated functional networks. In: Proceedings of the National Academy of Sciences of the United States of America. vol. 102, pp. 9673-9678.

Friston, K.J., Williams, S., Howard, R., Frackowiak, R.S., Turner, R., 1996. Movement- related effects in fMRI time-series. Magn. Reson. Med. 35, 346-355.

Gibson, C., Folley, B.S., Park, S., 2009. Enhanced divergent thinking and creativity in musicians: a behavioral and near-infrared spectroscopy study. Brain Cognit. 69, 162-169.

Greicius, M.D., Krasnow, B., Reiss, A.L., Menon, V., 2003. Functional connectivity in the resting brain: a network analysis of the default mode hypothesis. In: Proceedings of the National Academy of Sciences of the United States of America. vol. 100, pp. 253258.

Guilford, J.P., 1967. The Nature of Human Intelligence. McGraw-Hill Companies, New York.

Han, Y., Wang, J., Zhao, Z., Min, B., Lu, J., Li, K., He, Y., Jia, J., 2011. Frequencydependent changes in the amplitude of low-frequency fluctuations in amnestic mild cognitive impairment: a resting-state fMRI study. Neuroimage 55, 287-295.

Heausler, N.L., Thompson, B., 1988. Structure of the torrance tests of creative thinking. Educ. Psychol. Meas. 48, 463-468.

Heilman, K.M., Nadeau, S.E., Beversdorf, D.O., 2003. Creative innovation: possible brain mechanisms. Neurocase 9, 369-379.

Hickok, G., Poeppel, D., 2007. The cortical organization of speech processing. Nat. Rev. Neurosci. 8, 393-402.

Hjelmervik, H., Hausmann, M., Osnes, B., Westerhausen, R., Specht, K., 2014. Resting states are resting traits-an FMRI study of sex differences and menstrual cycle effects in resting state cognitive control networks. PLoS One 9, e103492.

Hong, Z., Ng, K.K., Sim, S.K., Ngeow, M.Y., Zheng, H., Lo, J.C., Chee, M.W., Zhou, J., 2015. Differential age-dependent associations of gray matter volume and white matter integrity with processing speed in healthy older adults. Neuroimage 123 , 42-50.

James, W., 1890. The Principles of Psychology. Holt, New York.

Jiang, L., Zuo, X.-N., 2015. Regional homogeneity a multimodal, multiscale neuroimaging marker of the human connectome. Neuroscientist 22, 486-505.

Jung, R.E., Segall, J.M., Bockholt, H.J., Flores, R.A., Smith, S.M., Chavez, R.S., Haier, R.J., 2010. Neuroanatomy of creativity. Human. Brain Mapp. 31, 398-409.

Kendall, M., Gibbons, J.D., 1990. Rank Correlation Methods Edward Arnold. Oxford University Press, Oxford.

Kiefer, M., Weisbrod, M., Kern, I., Maier, S., Spitzer, M., 1998. Right hemisphere activation during indirect semantic priming: evidence from event-related potentials. Brain Lang. 64, 377-408.

Kim, K.H., 2008. Meta-analyses of the relationship of creative achievement to both IQ and divergent thinking test scores. J. Creat. Behav. 42, 106-130.

Krug, R., Stamm, U., Pietrowsky, R., Fehm, H.L., Born, J., 1994. Effects of menstrual cycle on creativity. Psychoneuroendocrinology 19, 21-31.

Li, Y.-C., Chen, C.-C., Chen, J.-H., 2011. Impact of visual repetition rate on intrinsic properties of low frequency fluctuations in the visual network. PLoS One 6, e18954.

Liu, Y., Wang, K., Chunshui, Y., He, Y., Zhou, Y., Liang, M., Wang, L., Jiang, T., 2008. Regional homogeneity, functional connectivity and imaging markers of Alzheimer's disease: a review of resting-state fMRI studies. Neuropsychologia 46, 1648-1656.

Martín-Brufau, R., Corbalán, J., 2016. Creativity and Psychopathology: sex matters. Creativity Res. J. 28, 222-228.

Mayseless, N., Shamay-Tsoory, S., 2015. Enhancing verbal creativity: modulating creativity by altering the balance between right and left inferior frontal gyrus with tDCS. Neuroscience 291, 167-176.

Mell, J.C., Howard, S.M., Miller, B.L., 2003. Art and the brain The influence of frontotemporal dementia on an accomplished artist. Neurology 60, 1707-1710.

Murphy, K., Birn, R.M., Handwerker, D.A., Jones, T.B., Bandettini, P.A., 2009. The impact of global signal regression on resting state correlations: are anti-correlated networks introduced? Neuroimage 44, 893-905.

Oldfield, R.C., 1971. The assessment and analysis of handedness: the Edinburgh inventory. Neuropsychologia 9, 97-113.

Petersen, N., Kilpatrick, L.A., Goharzad, A., Cahill, L., 2014. Oral contraceptive pill use and menstrual cycle phase are associated with altered resting state functional connectivity. Neuroimage 90, 24-32.

Power, J.D., Barnes, K.A., Snyder, A.Z., Schlaggar, B.L., Petersen, S.E., 2012. Spurious but systematic correlations in functional connectivity MRI networks arise from subject motion. Neuroimage 59, 2142-2154.

Preckel, F., Wermer, C., Spinath, F.M., 2011. The interrelationship between speeded and unspeeded divergent thinking and reasoning, and the role of mental speed. Intelligence $39,378-388$

Rogers, T.T., Hocking, J., Noppeney, U., Mechelli, A., Gorno-Tempini, M.L., Patterson, K., Price, C.J., 2006. Anterior temporal cortex and semantic memory: reconciling findings from neuropsychology and functional imaging. Cogn. Affect. Behav. Neurosci. 6, 201-213.

Runco, M.A., Jaeger, G.J., 2012. The standard definition of creativity. Creativity Res. J. 24, $92-96$

Ryman, S.G., van den Heuvel, M.P., Yeo, R.A., Caprihan, A., Carrasco, J., Vakhtin, A.A. Flores, R.A., Wertz, C., Jung, R.E., 2014. Sex differences in the relationship between white matter connectivity and creativity. Neuroimage 101, 380-389.

Sato, J.R., Salum, G.A., Gadelha, A., Crossley, N., Vieira, G., Manfro, G.G., Zugman, A., Picon, F.A., Pan, P.M., Hoexter, M.Q., 2016. Default mode network maturation and psychopathology in children and adolescents. J. Child Psychol. Psychiatry 57, 55-64.

Schölvinck, M.L., Maier, A., Ye, F.Q., Duyn, J.H., Leopold, D.A., 2010. Neural basis of global resting-state fMRI activity. In: Proceedings of the National Academy of Sciences. vol. 107, pp. 10238-10243.

Seger, C.A., Desmond, J.E., Glover, G.H., Gabrieli, J.D., 2000. Functional magnetic resonance imaging evidence for right-hemisphere involvement in processing unusual semantic relationships. Neuropsychology 14, 361-369.

Shamay-Tsoory, S., Adler, N., Aharon-Peretz, J., Perry, D., Mayseless, N., 2011. The origins of originality: the neural bases of creative thinking and originality. 
Neuropsychologia 49, 178-185.

Shimonaka, Y., Nakazato, K., 2007. Creativity and factors affecting creative ability in adulthood and old age. Jpn. J. Educ. Psychol. 55, 231-243.

Silver, M., Montana, G., Nichols, T.E., 2012. False positives in neuroimaging genetics using voxel-based morphometry data. Neuroimage 54, 992-1000.

Simmons, W., Martin, A., 2009. The anterior temporal lobes and the functional architecture of semantic memory. J. Int. Neuropsychol. Soc. 15, 645-649.

Smith, S.M., Nichols, T.E., 2009. Threshold-free cluster enhancement: addressing problems of smoothing, threshold dependence and localisation in cluster inference. Neuroimage 44, 83-98.

Society_For_Creative_Minds, 1969. Manual of S-A Creativity Test. Tokyo shinri Corporation, Tokyo, Japan.

Song, M., Zhou, Y., Li, J., Liu, Y., Tian, L., Yu, C., Jiang, T., 2008. Brain spontaneous functional connectivity and intelligence. Neuroimage 41, 1168-1176.

Stein, M.I., 1953. Creativity and culture. J. Psychol. 36, 311-322.

Sternberg, R.J., 2005. Handbook of Creativity. Cambridge UP, New York.

Szameitat, A., Schubert, T., Müller, K., Von Cramon, D., 2002. Localization of executive functions in dual-task performance with fMRI. Cogn. Neurosci., J. 14, 1184-1199.

Takeuchi, H., Taki, Y., Hashizume, H., Sassa, Y., Nagase, T., Nouchi, R., Kawashima, R., 2011a. Cerebral blood flow during rest associates with general intelligence and creativity. PLoS One 6, e25532.

Takeuchi, H., Taki, Y., Hashizume, H., Sassa, Y., Nagase, T., Nouchi, R., Kawashima, R., 2011b. Failing to deactivate: the association between brain activity during a working memory task and creativity. Neuroimage 55, 681-687.

Takeuchi, H., Taki, Y., Hashizume, H., Sassa, Y., Nagase, T., Nouchi, R., Kawashima, R., 2011c. Failing to deactivate: the association between brain activity during a working memory task and creativity. Neuroimage 55, 681-687.

Takeuchi, H., Taki, Y., Hashizume, H., Sassa, Y., Nagase, T., Nouchi, R., Kawashima, R., 2012. The association between resting functional connectivity and creativity. Cereb. Cortex 22, 2921-2929.

Takeuchi, H., Taki, Y., Nouchi, R., Sekiguchi, A., Hashizume, H., Sassa, Y., Kotozaki, Y., Miyauchi, C.M., Yokoyama, R., Iizuka, K., Seishu, N., Tomomi, N., Kunitoki, K., Kawashima, R., 2015a. Degree centrality and fractional amplitude of low-frequency oscillations associated with stroop interference. Neuroimage 119, 197-209.

Takeuchi, H., Taki, Y., Nouchi, R., Yokoyama, R., Kotozaki, Y., Nakagawa, S., Sekiguchi, A., Iizuka, K., Yamamoto, Y., Hanawa, S., Araki, T., Miyauchi, C.M., Shinada, T., Sakaki, K., Sassa, Y., Nozawa, T., Ikeda, S., Yokota, S., Daniele, M., Kawashima, R., 2017. Creative females have larger white matter structures: evidence from a large sample study. Hum. Brain Mapp. 38, 414-430.

Takeuchi, H., Taki, Y., Sassa, Y., Hashizume, H., Sekiguchi, A., Fukushima, A., Kawashima, R., 2010a. Regional gray matter volume of dopaminergic system associate with creativity: evidence from voxel-based morphometry. Neuroimage 51, $578-585$.

Takeuchi, H., Taki, Y., Sassa, Y., Hashizume, H., Sekiguchi, A., Fukushima, A., Kawashima, R., 2010b. White matter structures associated with creativity: evidence from diffusion tensor imaging. Neuroimage 51, 11-18.

Takeuchi, H., Taki, Y., Sassa, Y., Hashizume, H., Sekiguchi, A., Fukushima, A., Kawashima, R., 2011d. Verbal working memory performance correlates with regional white matter structures in the fronto-parietal regions. Neuropsychologia 49, 3466-3473.

Takeuchi, H., Taki, Y., Thyreau, B., Sassa, Y., Hashizume, H., Sekiguchi, A., Nagase, T., Nouchi, R., Fukushima, A., Kawashima, R., 2013. White matter structures associated with empathizing and systemizing in young adults. Neuroimage 77, 222-236.

Takeuchi, H., Tomita, H., Taki, Y., Kikuchi, Y., Ono, C., Yu, Z., Sekiguchi, A., Nouchi, R., Kotozaki, Y., Nakagawa, S., 2015b. The associations among the dopamine D2 receptor Taq1, emotional intelligence, creative potential measured by divergent thinking, and motivational state and these associations' sex differences. Front. Psychol. 6, 912.

Tian, L., Ren, J., Zang, Y., 2012. Regional homogeneity of resting state fMRI signals predicts stop signal task performance. Neuroimage 60, 539-544.
Tomasi, D., Wang, G.-J., Volkow, N.D., 2013. Energetic cost of brain functional connectivity. In: Proceedings of the National Academy of Sciences. vol. 110, pp. 13642-13647.

Tononi, G., McIntosh, A.R., Russell, D.P., Edelman, G.M., 1998. Functional clustering: identifying strongly interactive brain regions in neuroimaging data. Neuroimage 7, $133-149$.

Torrance, E.P., 1966. Torrance tests of creative thinking. Scholastic Testing Service Bensenville, Ill, Bensenville, IL.

Treffinger, D.J., 1985. Review of the torrance tests of creative thinking. In: Mitchell, V.J.Jr (Ed.), The Ninth Mental Measurements Yearbook. University of Nebraska, Buros Institute of Mental Measurements, Lincoln, Nebraska, 1632-1634.

Vul, E., Harris, C., Winkielman, P., Pashler, H., 2009. Reply to comments on "puzzlingly high correlations in fMRI studies of emotion, personality, and social cognition". Perspect. Psychol. Sci. 4, 319-324.

Wang, L., Dai, W., Su, Y., Wang, G., Tan, Y., Jin, Z., Zeng, Y., Yu, X., Chen, W., Wang, X., 2012. Amplitude of low-frequency oscillations in first-episode, treatment-naive patients with major depressive disorder: a resting-state functional MRI study. PLoS One 7, e48658.

Wang, X., Xia, M., Lai, Y., Dai, Z., Cao, Q., Cheng, Z., Han, X., Yang, L., Yuan, Y., Zhang, Y., 2014. Disrupted resting-state functional connectivity in minimally treated chronic schizophrenia. Schizophr. Res. 156, 150-156.

Wang, Z., Yan, C., Zhao, C., Qi, Z., Zhou, W., Lu, J., He, Y., Li, K., 2011. Spatial patterns of intrinsic brain activity in mild cognitive impairment and alzheimer's disease: a resting-state functional MRI study. Human. Brain Mapp. 32, 1720-1740.

Watanabe, T., 1998. A study on the individual differences of the experience of hypnagogic imagery. Shinrigaku kenkyu: Jpn. J. Psychol. 68, 478-483.

Wei, D., Yang, J., Li, W., Wang, K., Zhang, Q., Qiu, J., 2013. Increased resting functional connectivity of the medial prefrontal cortex in creativity by means of cognitive stimulation. Cortex 51, 92-102.

Whitney, C., Kirk, M., O'Sullivan, J., Ralph, M.A.L., Jefferies, E., 2011. The neural organization of semantic control: tms evidence for a distributed network in left inferior frontal and posterior middle temporal gyrus. Cereb. Cortex 21, 1066-1075.

Xu, J., Rees, G., Yin, X., Song, C., Han, Y., Ge, H., Pang, Z., Xu, W., Tang, Y., Friston, K., 2014. Spontaneous neuronal activity predicts intersubject variations in executive control of attention. Neuroscience 263, 181-192.

Yan, C.-G., Cheung, B., Kelly, C., Colcombe, S., Craddock, R.C., Martino, A.D., Li, Q., Zuo, X.-N., Castellanos, F.X., Milham, M.P., 2013. A comprehensive assessment of regional variation in the impact of head micromovements on functional connectomics. NeuroImage 76, 183-201.

Yan, C.-G., Wang, X.-D., Zuo, X.-N., Zang, Y.-F., 2016. DPABI: data processing \& analysis for (Resting-State) brain imaging. Neuroinformatics, 1-13.

Yan, C., Zang, Y., 2010. DPARSF: a MATLAB toolbox for" pipeline" data analysis of resting-state fMRI. Front. Syst. Neurosci. 4, 13.

Yang, Z., Jutagir, D.R., Koyama, M.S., Craddock, R.C., Yan, C.-G., Shehzad, Z., Castellanos, F.X., Di Martino, A., Milham, M.P., 2015. Intrinsic brain indices of verbal working memory capacity in children and adolescents. Dev. Cogn. Neurosci. $15,67-82$.

Yao, Z., Wang, L., Lu, Q., Liu, H., Teng, G., 2009. Regional homogeneity in depression and its relationship with separate depressive symptom clusters: a resting-state fMRI study. J. Affect. Disord. 115, 430-438.

Zang, Y.-F., He, Y., Zhu, C.-Z., Cao, Q.-J., Sui, M.-Q., Liang, M., Tian, L.-X., Jiang, T., Wang, Y.-F., 2007. Altered baseline brain activity in children with ADHD revealed by resting-state functional MRI. Brain Dev. 29, 83-91.

Zang, Y., Jiang, T., Lu, Y., He, Y., Tian, L., 2004. Regional homogeneity approach to fMRI data analysis. Neuroimage 22, 394-400.

Zhang, X.-D., Cheng, Y., Poon, C.S., Qi, R.-f., Xu, Q., Chen, H.-J., Kong, X., Lu, G.-M., Shen, W., Zhang, L.-J., 2015. Long-and short-range functional connectivity density alteration in non-alcoholic cirrhotic patients one month after liver transplantation: a resting-state fMRI study. Brain Res.. 